\section{Toothpaste for naughty boys and girls}

Conventional fluoride toothpastes only deliver fluoride for a maximum of 90 minutes, whatever their fluoride content and provided the patient does not rinse. Biomin $\mathrm{F}$ toothpaste remains

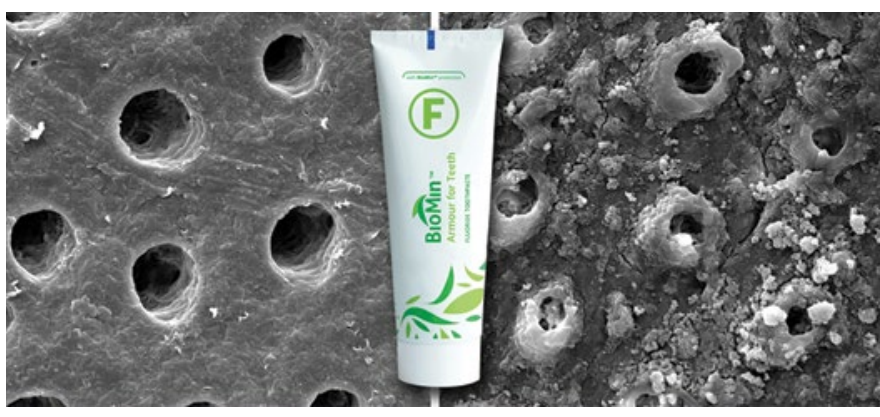

active for up to 12 hours, continuously releasing fluoride throughout this time to strengthen teeth and protect against decay. This applies even if the patient's toothbrushing technique is erratic and less efficient. Consequently, Biomin $\mathrm{F}$ is the perfect toothpaste for naughty boys and girls, whatever their age!

Available from Trycare, Biomin F utilises the latest bioglass technology to harden teeth against acid attack and reduce sensitivity, including scaling and post-bleaching sensitivity, a problem that reduces patient acceptance of this highly profitable revenue stream.

BioMin F contains tiny bioglass particles made up of fluoro calcium phosphosilicate bioactive glass which bonds to the teeth and enters the dentinal tubules, where they gradually dissolve over a period of up to 12 hours, slowly releasing calcium, fluoride and phosphate ions. These combine with saliva to form fluorapatite which strengthens the teeth, aids effective remineralisation of the enamel and prevents the fluid flow through the dentinal tubules which triggers sensitivity.

Patients also report that their teeth feel smoother and cleaner, that they notice an absence of background oral sensitivity and that their gums are healthier and less prone to bleeding.

BioMin $\mathrm{F}$ is the only toothpaste approved by the Oral Health Foundation for sensitivity relief and remineralisation.

Visit www.trycare.co.uk/biomin, contact your local representative or call 01274885544.

\section{Supreme performance}

Look no further than Clark Dental to offer you Sirona’s new XIOS XG Supreme intraoral sensor. It boasts CMOS-APS (Active Pixel Sensor) technology, enabling it to capture particularly low noise, high contrast images at an outstanding resolution of $33.3 \mathrm{lp} / \mathrm{mm}$.

The sensor's dynamic focus feature enables you to configure image sharpness, brightness and contrast for a range of diagnostic settings. This ultimately helps save time for future diagnostic procedures in general and restorative dentistry, as well as endodontics and periodontics.

Ideal for use in multiple treatment rooms, the XIOS XG Supreme transmits images in a matter of seconds for a more streamlined workflow.

For more information, call Clark Dental on 01268733 146, email info@clarkdental.co.uk or visit www.clarkdental.co.uk.

\section{Skip the hydration phase, reduce the risk}

Trycare Ltd have recently launched the new OsteoBiol GTO with TSV Gel.

Available in 0.5 and $2.0 \mathrm{cc}$ syringes, OsteoBiol GTO is Tecnoss's new ready-to-use pre-hydrated biomaterial that can be easily dispensed into defect sites direct from the syringe. It enables clinicians to skip the hydration phase with saline or blood, saving time and decreasing the risk of accidental exposure to pathogens. OsteoBiol GTO contains Tecnoss's innovative OsteoBiol TSV Gel which ensures optimal stickiness of the material, allowing easy adaptability to the recipient site and extreme stability.

Conceived as a universal biomaterial, easily adaptable to any bone defect, it is particularly indicated for horizontal augmentation of two-wall defects and socket preservation with compromised buccal plates. It can also be used in sinus lifting procedures where it can be applied directly through the bony window, helping to stabilise implants during immediate placement. It can also be used to treat peri-implant lesions and severe bony defects.

New OsteoBiol GTO with TSV Gel is the latest addition to the tried and trusted Tecnoss range which has been continuously developed for over 20 years.

For more information, call Trycare Ltd on 01274885544 or visit www.trycare.co.uk/osteobiol.

\section{Effective adhesion to all surfaces}

Dental adhesive should be versatile to your needs, easy to apply and provide a strong hold. After all, it's a bond that shouldn't be broken. Scotchbond universal dental adhesive from $3 \mathrm{M}$ Oral Care is made for effective adhering to all surfaces including enamel, dentine, glass ceramic, zirconia, noble and non-precious alloys and composites without the need for extra primer.

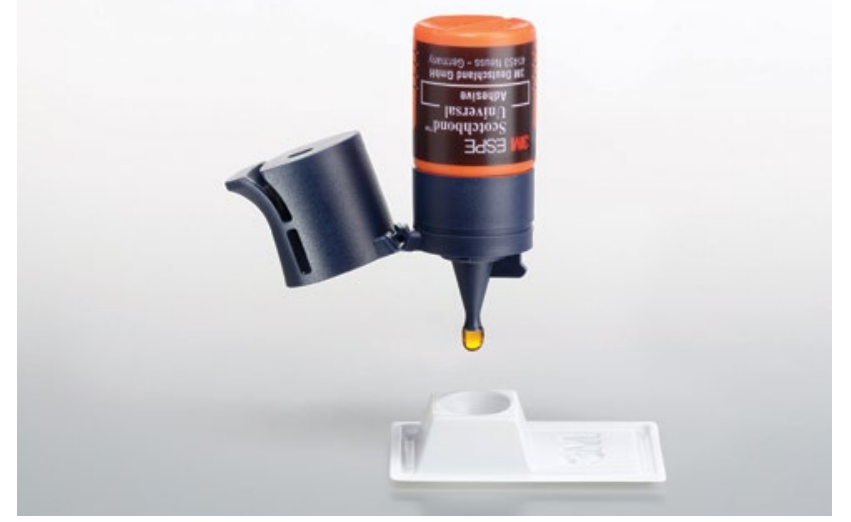

The adhesive is highly versatile and can also be applied using total-etch, self-etch or selective etch in both direct and indirect applications.

For more information, call 0800626578 or visit www.3M.co.uk/ Dental.

$3 \mathrm{M}$ and Scotchbond are trademarks of the 3M Company. 